\title{
Dissecting Seed Mucilage Adherence Mediated by FEI2 and SOS5
}

\author{
Jonathan S. Griffiths ${ }^{1 *}$, Marie-Jeanne Crepeau ${ }^{2}$, Marie-Christine Ralet ${ }^{2}$, \\ Georg J. Seifert ${ }^{3}$ and Helen M. North ${ }^{1}$
}

${ }^{1}$ Institut Jean-Pierre Bourgin, Institut National de la Recherche Agronomique, AgroParisTech, CNRS, Université Paris-Saclay, Versailles, France, ${ }^{2}$ Institut National de la Recherche Agronomique, UR 1268 Biopolymères Interactions Assemblages, Nantes, France, ${ }^{3}$ Department of Applied Genetics and Cell Biology, University of Natural Resources and Life Sciences, Vienna, Vienna, Austria

The plant cell wall is held together by the interactions between four major components: cellulose, pectin, hemicellulose, and proteins. Mucilage is a powerful model system to study the interactions between these components as it is formed of polysaccharides that are deposited in the apoplast of seed coat epidermal cells during seed development. When seeds are hydrated, these polysaccharides expand rapidly out of the apoplastic pocket, and form an adherent halo of mucilage around the seed. In Arabidopsis, mutations in multiple genes have similar loss of mucilage adherence phenotypes including CELLULOSE SYNTHASE 5 (CESA5)/MUCILAGE-MODIFIED 3 (MUM3), MUM5/MUCI21, SALT-OVERLY SENSITIVE 5 (SOS5), and FEI2. Here, we examine the interactions between these factors to better understand how they participate to control mucilage adherence. Double mutant phenotypes indicated that MUM5 and CESA5 function in a common mechanism that adheres pectin to the seed through the biosynthesis of cellulose and xylan, whereas SOS5 and FEI2, encoding a fasciclinlike arabinogalactan protein or a receptor-like kinase, respectively, function through an independent pathway. Cytological analyses of mucilage indicates that heteromannans are associated with cellulose, and not in the pathway involving SOS5 or FEI2. A SOS5 fluorescent protein fusion (SOS5-mCITRINE) was localized throughout the mucilage pocket, consistent with a structural role in pectin adhesion. The relationship between SOS5 and FEI2 mediated mucilage adherence was examined in more detail and while sos5 and fei2 mutants show similar phenotypes, key differences in the macromolecular characteristics and amounts of mucilage polymers were observed. FEl2 thus appears to have additional, as well as overlapping functions, with SOS5. Given that FEl2 is required for SOS5 function, we propose that FEI2 serves to localize SOS5 at the plasma membrane where it establishes interactions with mucilage polysaccharides, notably pectins, required for mucilage adherence prior to SOS5 being released into the apoplast.

Keywords: Arabidopsis, cell wall, mucilage, cellulose, arabinogalactan protein, receptor-like kinase, polysaccharides, genetics

\section{INTRODUCTION}

The plant cell wall is a complex structure composed of interconnected polysaccharides and proteins. Interactions between cell wall components determine cell shape, axis of elongation, and can affect permeability. Most primary cell walls are composed of three types of polysaccharides, cellulose, pectin, and hemicelluloses (Cosgrove, 2014). Primary plant cell walls are constantly 
reorganized to accommodate growth or to respond to changing environmental conditions. In order to correctly adjust to the external environment plant cells must be able to sense the conditions of the cell wall (Wolf et al., 2012). The specific mechanisms underlying this perception and response network are largely unknown.

Many receptor-like kinase genes (RLK) have been implicated as cell wall sensors. Multiple genes encoding wall-associated kinases (WAKs) are involved in cell wall signaling including THESEUS1, FEI1, and FEI2 (Hematy and Höfte, 2008; Xu et al., 2008; Höfte, 2015). FEI1 and FEI2 are transmembrane proteins that have an external leucine-rich repeat (LRR) domain, one transmembrane domain and a cytosolic kinase domain (Xu et al., 2008). In feil fei2 double mutants, there are substantial reductions in root cell expansion when grown in the presence of salt or sucrose (Xu et al., 2008). FEIland FEI2 have been proposed to be key components of a signaling pathway that monitors cell wall conditions, and can initiate an intracellular response through hormone signaling (Xu et al., 2008; Steinwand et al., 2014). The first step of this signaling pathway is believed to begin with the fasciclin-like arabinogalactan protein (FLA AGP), SALT-OVERLY SENSITIVE 5/FASCICLIN-LIKE ARABINOGALACTAN PROTEIN 4 (SOS5/FLA4; Shi et al., 2003; Xu et al., 2008). FLA AGPs are extracellular proteins that contain FASCICLIN (FAS) domains, are highly glycosylated, and often contain a C-terminal glycophosphatidyl inositol anchor (GPI; Johnson et al., 2003; Seifert and Roberts, 2007). Mutant sos 5 roots have a similar reduction in cell expansion as the feil fei2 double mutant, and a triple fei1 fei2 sos 5 mutant has a nonadditive phenotype, suggesting that these genes function together in a pathway regulating cell expansion (Xu et al., 2008). The precise mechanism through which FEIs and SOS5 control cell expansion remains unclear, with suggestions that these proteins might intervene directly in cellulose biosynthesis, or that they control cell wall architecture through pectin (Shi et al., 2003; Xu et al., 2008; Harpaz-Saad et al., 2011; Griffiths et al., 2014; Basu et al., 2015, 2016).

In addition to a role in modulating root cell shape, both FEI2 and SOS5 are required for the adherence of mucilage polysaccharides to the epidermal cells of the seed coat (Harpaz-Saad et al., 2011; Griffiths et al., 2014). Seed coat mucilage is formed from pectin-rich polysaccharides accumulated in the apoplast of the epidermal cell layer (Haughn and Chaudhury, 2005). These polysaccharides expand rapidly upon hydration of mature seeds, and form non-adherent and adherent layers in Arabidopsis. The adherent mucilage layer can only be removed with harsh acid treatment, or enzymatic digestion of pectin and cellulose (Macquet et al., 2007). Cellulose synthesized by CELLULOSE SYNTHASE 5 (CESA5), CESA3, and COBRA-LIKE 2 (COBL2) is required for mucilage adherence through xylans synthesized by MUCILAGE-MODIFIED 5/MUCILAGE RELATED 21 (MUM5/MUCI21), IRREGULAR XYLEM 7 (IRX7), and IRX14 and pectins (Mendu et al., 2011; Sullivan et al., 2011; Ben-Tov et al., 2015; Griffiths et al., 2015; Voiniciuc et al., 2015b; Hu et al., 2016a,b; Ralet et al., 2016). SOS5 and FEI2 mutants have a reduction in pectic-mucilage adherence similar to cesa5, yet how these two genes mediate adherence is still unknown (Harpaz-Saad et al., 2011; Griffiths et al., 2014).

When visualizing cellulose in the adherent mucilage layer, the distribution of cellulose can be divided into two distinct regions; an intensely staining ray-like structure located above the central columella of each cell, and a diffuse staining region between the rays (Macquet et al., 2007; Griffiths et al., 2014, 2015). Mutation of cesa5, mum5, and cobl2 results in a loss of mucilage adherence and staining of regions of diffuse cellulose in the adherent layer is lost while cellulose observed in rays appears to be mostly intact (Mendu et al., 2011; Sullivan et al., 2011; Ben-Tov et al., 2015; Voiniciuc et al., 2015b; Ralet et al., 2016). Although sos5 and fei2 mutants have a similar loss of pectin-mucilage adherence, they have an inverse effect on cellulose structure within the adherent layer; the ray structure is completely absent yet the diffuse staining region remains (Harpaz-Saad et al., 2011; Griffiths et al., 2014). The phenotypes of cesa5 and sos 5 seeds are clearly different and a cesa 5 sos 5 double mutant has a more severe phenotype than either single mutant, demonstrating that these two genes function independently to mediate mucilage adherence (Griffiths et al., 2014).

While the mechanism behind the attachment of mucilage pectin to CESA5 synthesized cellulose requires xylan branches on RG-I synthesized by MUM5 (Ralet et al., 2016), the mechanism underlying SOS5 and FEI2 mediated mucilage adherence remains unresolved (Griffiths et al., 2014). Here, we further investigate the role of SOS5 and FEI2 in mucilage adherence using a combination of genetics, polysaccharide chemistry, and localization of the SOS5 protein. We define two unique genetic pathways that mediate mucilage adherence, one through cellulose and another through SOS5-FEI2.

\section{MATERIALS AND METHODS}

\section{Plant Material}

The mutants cesa5-2 (SALK_099008), mum5-2 (WiscDSLox line 503F10), and sos5-2 (SALK_125874) are in the Col-0 accession (Xu et al., 2008; Sullivan et al., 2011; Ralet et al., 2016). The T-DNA insertion line SAIL 150_A08, in the Col-0 accession, was identified in the SIGnAL database (Alonso et al., 2003 ${ }^{1}$ ), seeds were obtained from the Nottingham Arabidopsis Stock Centre $^{2}$ and a homozygous line was identified which we named fei2-3. Double mutants for cesa5-2 fei2-3, mum5-2 cesa5-2, sos52 mum5-2, and sos5-2 fei2-3 were identified from F2 plants generated from crosses between the two respective single mutants by PCR using the primers listed in Supplementary Table 1, in combination with the insert specific primer LBb1.3 for SALK lines, and SAIL LB1 for SAIL lines (Table 1). Seeds were produced in a glasshouse $\left(18-28^{\circ} \mathrm{C}\right)$, with a minimum photoperiod of $13 \mathrm{~h}$ provided by supplemental lighting. Plants were grown in compost (Tref substrates) and watered with Plant-Prod nutritive solution $\left(\right.$ Fertil $\left.{ }^{3}\right)$. As the seed coat is a maternal tissue the F1 and F2

\footnotetext{
${ }^{1}$ http://signal.salk.edu

${ }^{2}$ http://arabidopsis.info

${ }^{3}$ http://www.plantprobes.net/index.php
} 
TABLE 1 | Sugar composition of adherent mucilage from wild-type, cesa5-2, sos5-2, and fei2-3 extracted with RGase, following water extraction.

\begin{tabular}{lllll}
\hline & Wild-type & cesa5-2 & sos5-2 & fei2-3 \\
\hline Rha & $3.08(0.17)$ & $1.41(0.14)^{*}$ & $1.38(0.09)^{*}$ & $1.37(0.08)^{*}$ \\
Fuc & $0.07(0.01)$ & $0.06(0.004)$ & $0.10(0.03)$ & $0.10(0.05)$ \\
Ara & $0.16(0.009)$ & $0.17(0.02)$ & $0.15(0.009)$ & $0.19(0.02)$ \\
Xyl & $0.10(0.01)$ & $0.09(0.01)$ & $0.09(0.01)$ & $0.14(0.08)$ \\
Man & $0.21(0.01)$ & $0.24(0.02)$ & $0.16(0.02)$ & $0.22(0.01)$ \\
Gal & $1.41(0.05)$ & $1.47(0.06)$ & $1.07(0.31)$ & $1.67(0.13)^{*}$ \\
Glu & $6.51(0.18)$ & $6.48(0.14)$ & $5.93(0.20)$ & $7.96(0.26)^{*}$ \\
\hline
\end{tabular}

Values in parentheses are SE of three independently extracted samples from three biological repeats. Asterisks indicate a significant difference from wild-type (MannWhitney $U$ test, $p<0.001$.

seed mucilage phenotypes were determined using the F2 and F3 seed, respectively. For all comparisons, seed lots used were obtained from plants that had been simultaneously cultivated and harvested.

\section{Cloning of proUBQ10:SOS5-mCitrine}

The proUBQ10:SOS5-mCitrine construct is based on pGREEN179 (GenBank: EUO48866.1; Hellens et al., 2000) plant transformation vector and contains PCR fragments of the $611 \mathrm{bp}$ promoter region including the $5^{\prime}$ UTR of UBQ10 (At4g05320), the 27 amino acid (aa) residue secretion signal of SOS5/FLA4, a SRVPV linker, mCitrine (Shaner et al., 2005; GenBank : AEJ82308.1; aa residues 1-239), followed by most of the SOS5/FLA4 coding region (At3g46550; aa residues 29-420) and the HSP18.2 (At5g59720) terminator (Nagaya et al., 2010). All primers used for cloning are shown in Supplementary Table 1.

\section{Cytochemical Staining and Immunolabeling Procedures}

Ruthenium red staining was performed as previously described (Sullivan et al., 2011). For cellulose staining, seeds were imbibed and shaken in water for $1 \mathrm{~h}$, followed by staining for $1 \mathrm{~h}$ with $0.01 \%$ (w/v) Direct Red 23 (DR; Sigma-Aldrich) in a $100 \mathrm{mM}$ $\mathrm{NaCl}$ solution ( $\mathrm{pH}$ 5.0). Seeds were rinsed then imaged on a Leica SP5 II AOBS confocal microscope with a Tandem HyD detector (561 nm excitation and 570-660 nm emission). Whole mount immunolabeling procedures were performed as described in Voiniciuc et al. (2015a) with LM14 and LM21 antibodies ${ }^{4}$ then imaged on a Leica SP5 II AOBS confocal microscope with a Tandem HyD detector (488 nm excitation and 500-550 nm emission for AlexaFluor 488 and $561 \mathrm{~nm}$ excitation and 570$660 \mathrm{~nm}$ emission for DR).

For imaging birefringence of polarized light, seeds were imbibed in water for $20 \mathrm{~min}$, mounted on a glass slide and observed using a Nikon SMZ800 binocular loop, a Nikon Coolpix E4500 camera and a Nikon simple polarized light adaptor (Nikon MNN40920 ${ }^{5}$.

\footnotetext{
${ }^{4} \mathrm{http}: / /$ www.plantprobes.net/index.php

${ }^{5}$ https://www.nikoninstruments.com/it_IT/
}

Seeds expressing proUBQ10:SOS5-mCitrine were excised from developing siliques at approximately 10 days post-anthesis (dpa). Seeds were stained with $50 \mu \mathrm{M}$ FM4-64 (Invitrogen) for $0.5 \mathrm{~h}$ then imaged on a Leica SP5 II AOBS Tandem HyD microscope with an excitation/emission of 516/525-575 nm for mCitrine, and 516/600-700 nm for FM4-64. All images were processed using ImageJ version $1.47 \mathrm{v}$ (Freeware, National Institutes of Health, $\left.\mathrm{USA}^{6}\right)$.

\section{Extraction and Analysis of Mucilage}

Analysis of the monosaccharide composition of mucilage was performed as detailed in Sullivan et al. (2011). Briefly, $200 \mathrm{mg}$ of seeds were hydrated in $4 \mathrm{~mL}$ of distilled water and shaken headover-tail for $3 \mathrm{~h}$. The supernatants were collected and filtered through a disposable glass microfiber filter $(13 \mathrm{~mm}$ diameter, $2.7 \mu \mathrm{m}$ pore size; Whatman ${ }^{7}$ ). Seeds were then rinsed twice, and rinses were discarded. The adherent mucilage layer was digested by adding 0.1 nkat of rhamnogalacturonan hydrolase (Swiss-Prot Q00018) provided by Novozymes ${ }^{8}$ in $4 \mathrm{~mL}$ of $50 \mathrm{mM}$ sodium acetate, $\mathrm{pH}$ 4.5. Seeds were incubated with the enzyme for $16 \mathrm{~h}$ at $40^{\circ} \mathrm{C}$, then centrifuged $(8000 \times \mathrm{g}, 3 \mathrm{~min})$, and the supernatant was collected and filtered through a disposable glass microfiber filter. Seeds were washed again three times with $5 \mathrm{~mL}$ of $50 \mathrm{mM}$ sodium acetate buffer, $\mathrm{pH} 4.5$, then treated with 0.9 nkat of Maxazyme $^{\circledast}$ cellulase (DSM, Seclin, France) at $40^{\circ} \mathrm{C}$ for $16 \mathrm{~h}$, before centrifuging $(8000 \times g, 3 \mathrm{~min})$.

Total uronic acid (GalA) and neutral sugar contents were determined with the automated $m$-hydroxybiphenyl and orcinol methods, respectively (Thibault, 1979; Tollier and Robin, 1979). Individual neutral sugars were analyzed as alditol acetate derivatives (Blakeney et al., 1983) by gas-liquid chromatography (Perkin Elmer Gas chromatograph) after hydrolysis with $2 \mathrm{M}$ trifluoroacetic acid at $121^{\circ} \mathrm{C}$ for $2.5 \mathrm{~h}$. Sugar amounts from different mutant lines were compared using the Mann-Whitney $U$ test to determine statistically significant differences.

For HP-SEC analysis, $200 \mathrm{mg}$ of seeds were hydrated in $4 \mathrm{~mL}$ of distilled water, and shaken head-over-tail for $3 \mathrm{~h}$. The supernatant was extracted following centrifugation $(8000 \times g$, $3 \mathrm{~min}$ ) filtered through a disposable glass microfiber filter, and boiled for $5 \mathrm{~min}$. HP-SEC analysis of water-extracted mucilage was performed at room temperature with a Shodex OH SBG precolumn and a Shodex OH-Pack SB-805 HQ column'. Elution was carried out with $50 \mathrm{mM}$ sodium nitrate buffer at a constant flow rate of $60 \mathrm{~mL} \mathrm{~h}^{-1}$. Polymers were detected with a differential refractometer (VE 3580 RI detector) and a Viscotek 270 Dual Detector (dual laser light scattering, wavelength $=670 \mathrm{~nm}, 90^{\circ}$ and $7^{\circ}$ combined with a differential pressure viscometer (Malvern Instruments ${ }^{10}$ ). Detectors were calibrated with a pullulan standard with a narrow molecular mass distribution $\left(M \mathrm{w}=145618 \mathrm{D}, M_{\mathrm{n}}=139180 \mathrm{D},[\eta]=54 \mathrm{~mL} \mathrm{~g}^{-1}\right.$ at $30^{\circ} \mathrm{C}$ in $0.1 \mathrm{M}$ sodium nitrate, $\mathrm{dn} / \mathrm{dc}=0.147 \mathrm{~mL} \mathrm{~g}^{-1}$; Malvern

\footnotetext{
${ }^{6}$ https://imagej.nih.gov/ij/

${ }^{7}$ http://www.whatman.com/index.aspx

${ }^{8}$ http://www.novozymes.com/

${ }^{9}$ http://www.shodex.de/

${ }^{10} \mathrm{http}: / /$ www.malvern.com/
} 
Instruments). Samples were automatically injected through a $50 \mu \mathrm{L}$ loop. Data analysis was performed using OmniSec version 4.5 software (Malvern Instruments).

\section{RESULTS}

\section{Both FEI2 and SOS5 Mediate Mucilage Adherence through a Common Pathway that Is Independent of Cellulose}

Cellulose synthase 5 and SOS5 have previously been shown to mediate mucilage adherence through two distinct and independent mechanisms (Griffiths et al., 2014). In order to clarify the relationship between CESA5, SOS5, and other proteins involved in mucilage adherence, the pectin and cellulose that form the adherent mucilage were examined in single and double mutants. Ruthenium red (RR) stained mutant seeds of cesa5-2, sos5-2, mum5-2, and a new mutant allele of FEI2, fei2-3 (SAIL 150_A08), were compared to wild-type. All four had little RR staining of pectin as previously described (Figure 1A; HarpazSaad et al., 2011; Mendu et al., 2011; Sullivan et al., 2011; Griffiths et al., 2015; Voiniciuc et al., 2015b; Ralet et al., 2016). Nevertheless, sos5-2 and fei2-3 seeds often had larger unstained halos surrounding seeds, which was rarely observed for cesa52 or mum5-2 seeds (Figure 1). When stained for cellulose with direct red (DR), two regions can be distinguished in wildtype mucilage, a central ray region (see arrow, Figure 1) above the columella (see asterisks, Figure 1), and a diffuse staining region around the rays. While cesa5-2 and mum5-2 seeds still had observable ray structures (see arrows, Figures 1B,C), these were no longer present in sos5-2 and fei2-3 mucilage. The latter mutants still had diffusely stained cellulose, whilst this was absent in cesa5 and mum5 (Figures 1B,C). We can therefore expand on the previously elucidated independent mechanisms for mucilage attachment by CESA5 and SOS5 (Griffiths et al., 2014) to define two distinct pathways mediating pectin-mucilage adherence based on cellulose staining. First, MUM5 is involved in the pathway required for adherence through cellulose synthesized by CESA5, which is observed as a diffuse staining region within mucilage. A second pathway requires FEI2 and SOS5 for the formation of cellulosic rays. These two pathways are clearly delineated by the staining patterns of cellulose in the adherent mucilage layer.

To confirm these pathways genetically, double mutants between the different components mediating mucilage adherence were generated and examined. The RR stained halo of mucilage observed in the cesa5-2 mum5-2 double mutant was similar to cesa5-2 and mum5-2 single mutants (Figure 1D) and did not represent an additive phenotype. Furthermore, rays of cellulose were still visible in the cesa5-2 mum5-2 double mutants (see arrow, Figures 1E,F) confirming that CESA5 and MUM5 are involved in a common mechanism that mediates mucilage adherence and the formation of the diffusely stained cellulose regions. In contrast, sos5-2 mum5-2 seeds exhibited a greater reduction in pectin to that observed in the mum5-2 and sos5-2 single mutants and there was no staining of either rays or diffuse regions in double mutant mucilage (Figures 1D-F). This was similar to the additive phenotype previously reported for cesa5-2 sos5-2 (Figures 1D-F), (Griffiths et al., 2014) in accord with SOS5 being involved in a mechanism independent of the attachment of mucilage pectin to cellulose through xylan. Similarly, a drastic reduction in $\mathrm{RR}$ staining and an absence of cellulose staining were observed for cesa5-2 fei2-3 mucilage (Figures 1D-F) suggesting that FEI2 also affects mucilage adherence through a mechanism independent of CESA5. To determine whether this could be the same as that for SOS5 a sos5-2 fei2-3 mutant was generated. The mucilage of double mutant seeds did not appear to be more severely affected than either single mutant, with diffuse staining of cellulose still observed in the adherent layer (Figures 1D,E). Taken together these results demonstrate that in the two independent pathways that mediate mucilage adherence, one requires cellulose synthesized by CESA5 and xylans synthesized by MUM5, and the second comprises SOS5 and FEI2.

To further examine the role of SOS5 and FEI2 in cellulose mediated mucilage adherence, polarized light was used to examine crystalline cellulose in the adherent mucilage layer of cesa5-2, sos5-2, fei2-3, sos5-2 fei2-3, and cesa5-2 fei2-3 seeds (Figure 2). When hydrated in water and viewed under polarized light, the mucilage of wild-type seeds was seen as a bright halo, while no reflection of polarized light was observed in cesa5-2 mucilage (Figure 2). Interestingly, the adherent mucilage of both sos5-2 and fei2-3 seeds still displayed birefringent halos, although these were smaller and exhibited some curious differences. The reflection of light was reduced in fei2-3 and sos5-2 fei2-3 seeds compared to wild-type or sos5-2 seeds (Figure 2). Finally, cesa52 fei2-3 birefringent halos were similar to cesa5-2 seeds, and reduced compared to fei2-3 seeds, in agreement with FEI2 function being independent of CESA5. These results also suggest that fei2-3 seeds have a more severe phenotype compared to sos5-2 seeds, indicating a function for FEI2 independent of that of SOS5 that could be involved in determining the degree of cellulose crystallinity in adherent mucilage.

\section{Heteromannan Structuring of Cellulose Appears to Be Independent of the SOS5-FEI2 Pathway}

Reductions in birefringence of crystalline cellulose have also been observed for other components involved in adherent mucilage structure, notably two genes that synthesize heteromannans, CSLA2 and MUCI10 (Yu et al., 2014; Voiniciuc et al., 2015a). Since FEI2 has a similar reduction in crystalline cellulose birefringence and also appears to function independently of CESA5, we investigated the relationship between CESA5, SOS5, and FEI2 with heteromannan through immunolabeling with the LM21 antibody (Marcus et al., 2010). The LM21 signal is relatively weak in wild-type seeds, but extensive labeling of the adherent mucilage halo can be observed, although rays are not strongly labeled by LM21 (Figures 3A,B; Voiniciuc et al., 2015b). In contrast cesa5-2 seeds had a strong LM21 signal, which was much more intense than that of wild-type, and was also colocalized with rays indicating a link between production 


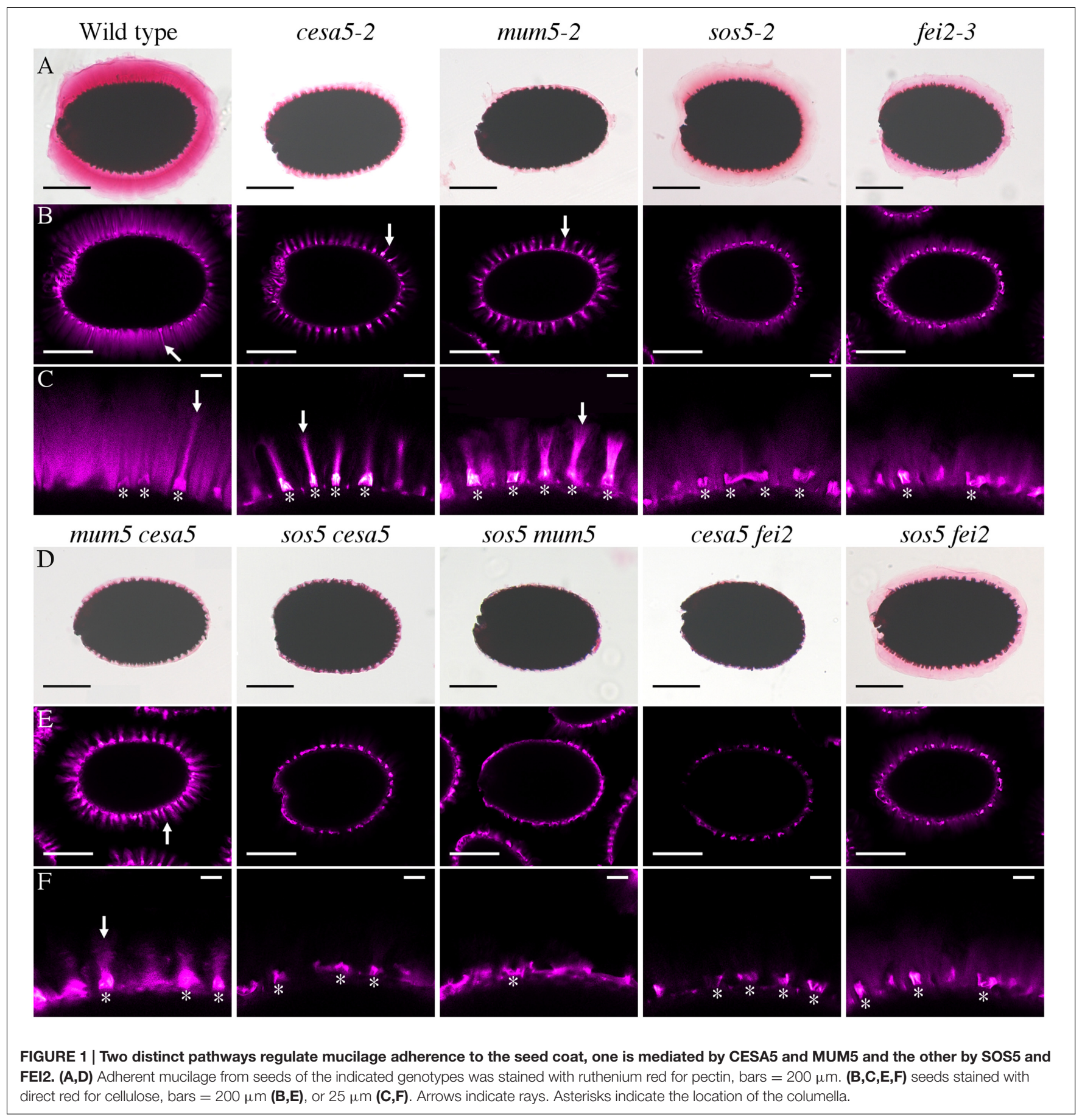

of cellulose and heteromannan (see arrow, Figure 3B). The localization of LM21 signal in sos5-2, fei2-3, and sos5-2 fei2-3 mucilage was the inverse of that observed for cesa5-2 seeds, with labeling being particularly intense in mucilage regions situated above the radial walls, corresponding to the interphase between the mucilage released from two independent epidermal cells (see asterisks, Figure 3B); the sos5-2 fei2-3 double mutant was indistinguishable from either single mutant. The localization of LM21 signal in sos5-2 and fei2-3 mucilage was consistent with a general loss of mucilage adherence and faulty ray organization, indicating that SOS5 and FEI2 function independently of heteromannan in mediating mucilage adherence and cellulose crystallinity.

We also examined the localization of LM14, an antibody specific to arabinogalactan proteins (Moller et al., 2008) to try to understand the localization of SOS5, and the effects of CESA5 and FEI2 on SOS5 localization. In wild-type seeds, LM14 signal appeared to be more specific to the interphase between mucilage 


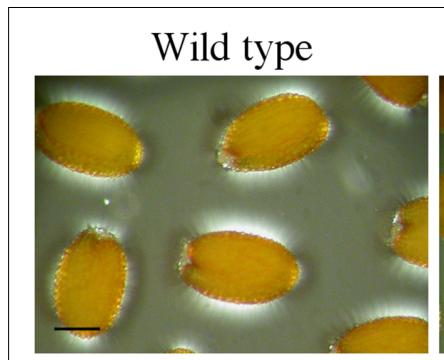

$\operatorname{sos} 5-2$

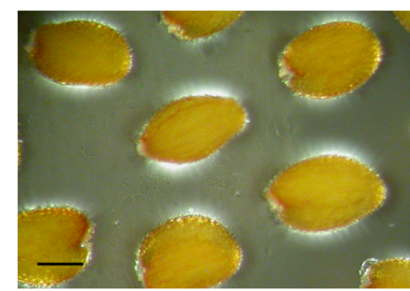

sos 5 fei2

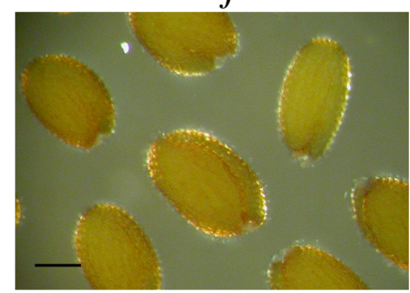

FIGURE 2 | Polarized light birefringence of crystalline cellulose microfibrils is not affected in sos5-2 seeds. Imbibed seeds were visualized under polarized light. Bars $=200 \mu \mathrm{m}$. released from two different epidermal cells, and it did not label rays (Figures 3C,D). In cesa5-2 mucilage, LM14 was specifically localized around the ray, and appears to coat the base of the ray, yet the signal did not colocalize with the DR stain of cellulose (see arrow, Figure 3). The localization of LM14 in cesa5-2 seeds is very similar to that of cesa5-1 seeds labeled with the RG-I specific antibody CCRC-M36 (Griffiths et al., 2014, 2015), which suggests an association between arabinogalactan proteins and RG-I, potentially serving to organize the ray. In sos $5-2$ and sos 5 fei2 double mutant seeds, LM14 signal was localized around the columella, with some signal observable above the columella despite the lack of a cellulosic ray (see arrow, Figure 3). Similar to wild-type seeds, clear signals were observed in the interphase region of mucilage released from two different cells in sos5-2, fei23 , and sos5-2 fei2-3 seeds. The arabinogalactan proteins labeled by LM14 in the mucilage are not, therefore, uniquely SOS5, which suggests that other arabinogalactan proteins are involved in structuring adherent mucilage.

\section{FEI2 Has a Predominant Role in Mucilage Adhesion Compared to SOS5}

To gain more information concerning the mechanism by which SOS5 and FEI2 intervene in mucilage adherence a detailed analysis of adherent mucilage composition was carried out. Mucilage was extracted sequentially with water from the same weight of seeds for each genotype followed by digestion of the inner mucilage layer with rhamnogalacturonan hydrolase (RGase). Finally, cellulase was used to hydrolyse the cellulose still attached to the seed coat. Following the water extraction differences in the volume of the seeds and remaining adherent mucilage were observed compared to wild-type (Figure 4A). Wild-type seeds had the greatest volume, followed by sos5-2 seeds, while cesa5-2 and fei2-3 seeds occupied the smallest volume (Figure 4A). Following RGase treatment, however, all three mutants had a similarly reduced volume compared to wild-type (Figure 4B). Finally, after cellulase treatment the seed volume for all four genotypes was equivalent (Figure 4C). The difference in seed volume for sos5-2 and fei2-3 seeds prior to RGase treatment again indicated that fei2-3 has a stronger effect on mucilage adherence than sos5-2.

The total amounts of acid sugars extracted with water or following RGase digestion were quantified (Figure 4D), and the amounts of individual monosaccharides were determined in RGase hydrolysates (Table 1). Consistent with their loss of mucilage adherence phenotype, cesa5-2, sos5-2, and fei2-3 seeds had increased amounts of sugars extracted from non-adherent mucilage, and reduced amounts from adherent mucilage, as previously reported (Harpaz-Saad et al., 2011; Mendu et al., 2011; Sullivan et al., 2011; Griffiths et al., 2014); amounts of Rha in RGase extracts were significantly lower in all three mutants compared to wild-type (Table 1; Mann-Whitney $U$ test, $p<0.001)$. Interestingly, sos5-2 seeds had more GalA in RGase hydrolysates than cesa5-2 or fei2-3 (Figure 4D; Mann-Whitney

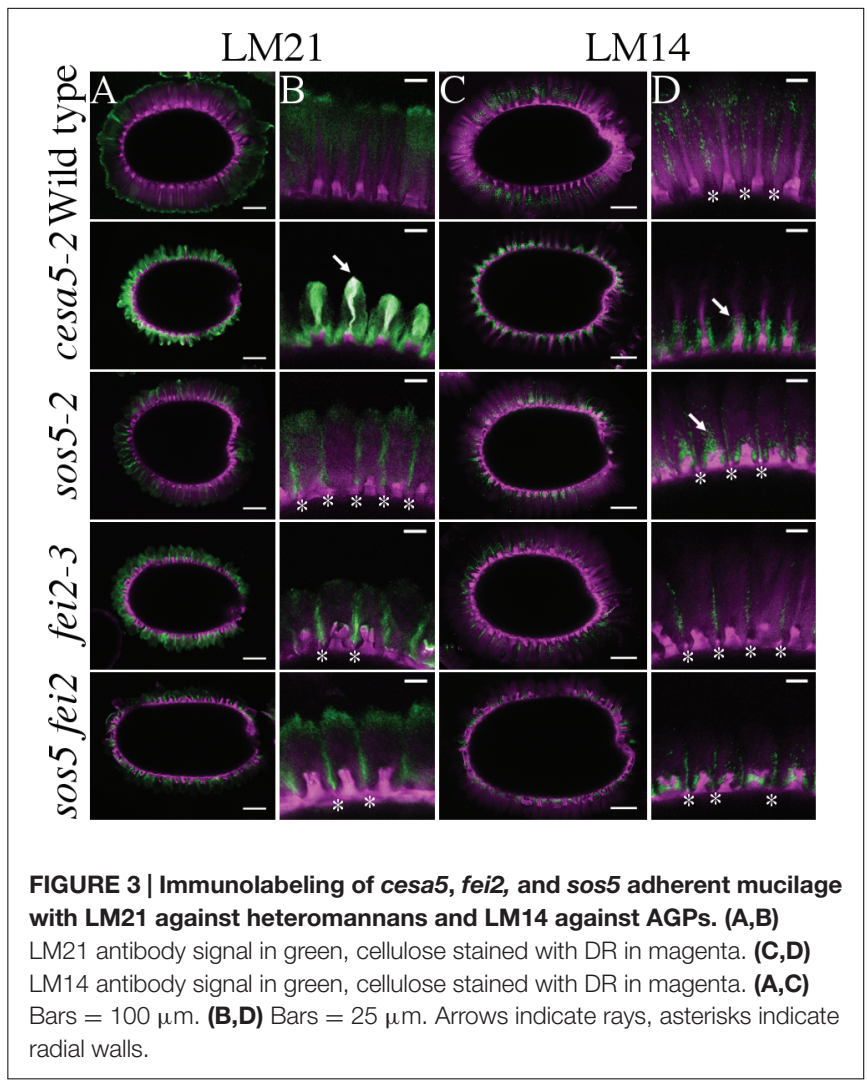




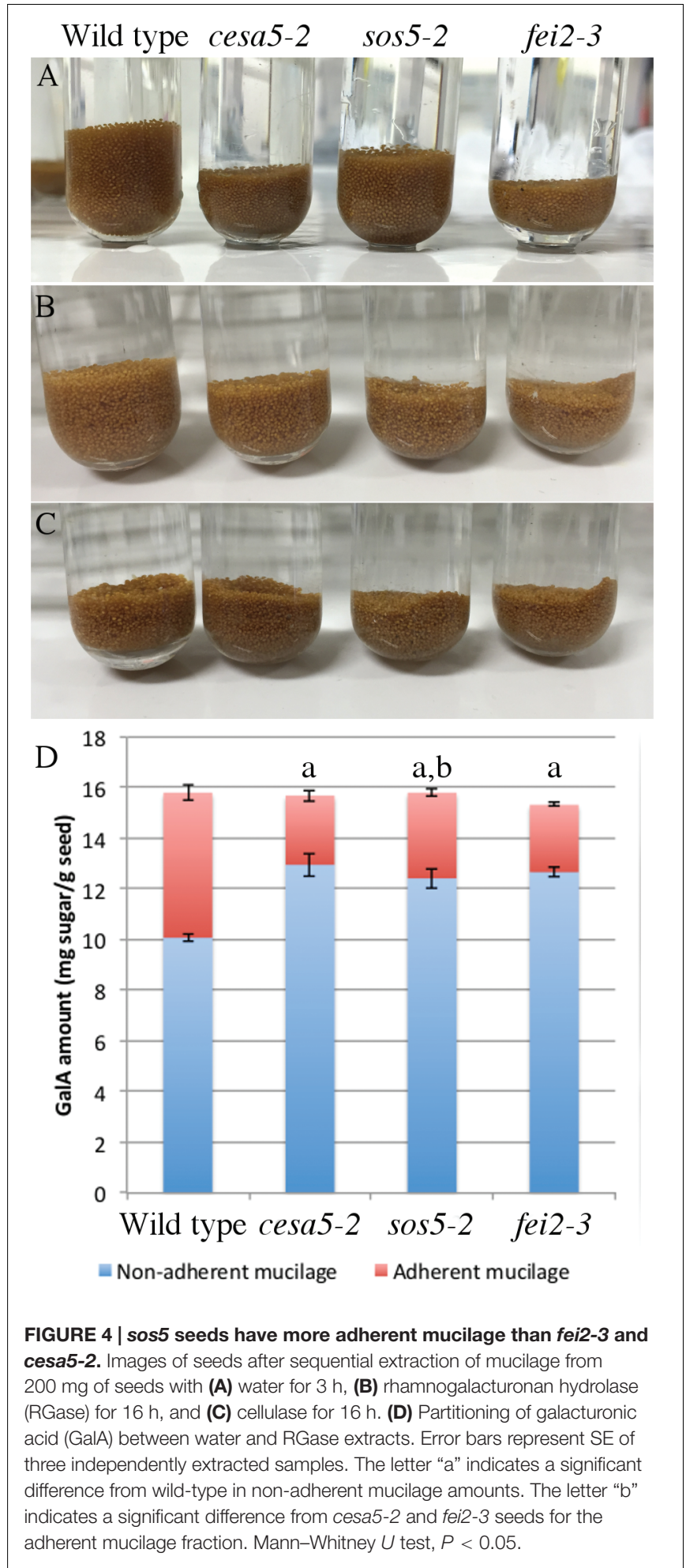

$U$ test, $p<0.05)$, in accord with the larger volume of their seeds and adherent mucilage after water extraction alone (Figure 4A). This confirmed that sos5-2 mutation has a weaker effect on mucilage adherence than defects in the other genes. Together with the images of birefringence, these results demonstrate that in addition to differences between CESA5 and the SOS5-FEI2 pathway in mediating adherence the overlap between the roles of SOS5 and FEI2 is not complete and suggest that FEI2 has additional functions beyond SOS5.

To examine whether the presence of RG-I is responsible for the organization of cellulose into two differently staining populations, seeds were stained with DR following RGase digestion. Brightly stained cellulose was still observed in the adherent mucilage of wild-type seeds as both rays and diffuse regions (Figure 5). Furthermore, no modification was observed in the structure of the cellulose observed in the mutants with cesa5-2 mucilage still having cellulose in rays, while sos5-2 and fei2-3 cellulose still appeared diffuse (Figure 5). These results suggest that the presence of rays does not require RG-I, and that rays could be pre-determined in the mucilage pocket.

The macromolecular characteristics of mucilage polysaccharides extracted with water from mutant seeds were examined using HP-SEC to obtain information about how SOS5 and FEI2 affect polysaccharide conformation and interactions. Wild-type mucilage separated into two distinct polymeric fractions, an initial small peak representing large molecular weight polymers, and a second larger peak representing relatively smaller molecular weight polymers (Figure 6; Table 2; Macquet et al., 2007). cesa5 mutant seeds lacked the large molecular weight polymeric fraction (Figure 6; Sullivan et al., 2011). Comparable results have also been observed for irx7, irx14, and mum5 (Macquet et al., 2007; Hu et al., 2016a,b). In contrast, both sos5-2 and fei2-3 extracts retained the first peak, showing that the larger polymeric aggregates are present in the mucilage of these mutants (Figure 6), and were more abundant in sos52, compared to wild-type. There was, however, a significant reduction in both the weight-average and the number-average molecular weights ( $M_{\mathrm{w}}$ and $M_{\mathrm{n}}$, respectively) in sos5-2 and fei2-3 polymers, compared to wild-type (Table 2), the polydispersity
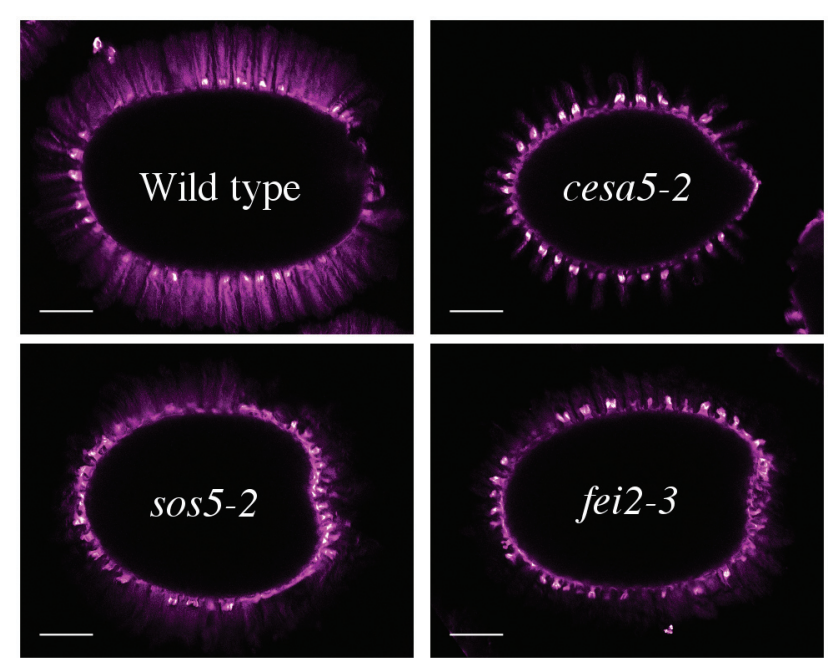

FIGURE 5 | Structure of cellulose in adherent mucilage after digestion with rhamnogalacturonan hydrolase for $\mathbf{1 6} \mathbf{~ h}$. Cellulose was stained with direct red. Bar $=200 \mu \mathrm{m}$. 


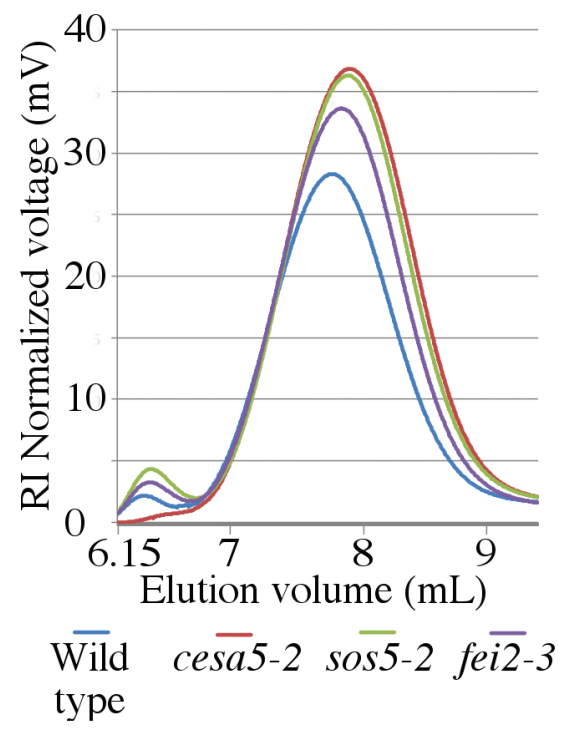

FIGURE 6 | Water-extracted mucilage from sos5 and fei2 has distinct macromolecular properties compared to wild-type and cesa5. Water extracted mucilage was separated by size-exclusion chromatography combined with refractive index detection. Plots are the average value of three independent biological replicates. index $\left(M_{\mathrm{w}} / M_{\mathrm{n}}\right)$ being further from 1 . This population of RG-I polymers in sos5-2 and fei2-3 mucilage had, therefore a greater range of sizes, and were on average smaller than in the wild-type. The intrinsic viscosity of sos5-2 and fei2-3 was also reduced compared to wild-type in this first polymeric fraction (Table 2), in accord with the reduced average polymer size. In contrast, the second polymeric fraction peaked at higher values in all three mutants compared to wild-type (Figure 6), with a significantly increased peak surface for cesa5-2 and sos5-2 (Table 2), in accord with the increased amount of mucilage extracted with water in these mutant seeds. In this second polymeric fraction polymers were shorter in the mutants compared to wild-type, being particularly marked for cesa5-2. Finally, cesa5-2 and sos5-2 had reduced intrinsic viscosity compared to wild-type mucilage. These results demonstrate that there are clear alterations to the macromolecular properties of the polysaccharides with a tendency toward smaller and likely shorter RG-I polymers in the mutants, which is most severe in cesa5-2. Furthermore, the mucilage polymers extracted with water from sos5-2 and fei2-3 seeds differ from those of cesa5-2 as they retain the population of high average molecular weight. This difference in SEC profile is in agreement with SOS5 and FEI2 having a function that is largely independent of cellulose.

\section{SOS5 Is Localized throughout the Mucilage Pocket}

In roots the SOS5-FEI2 pathway has been proposed to intervene in cell wall sensing and the initiation of a signaling cascade involving hormones. The SOS5 protein is an extracellular protein and has a GPI domain that could anchor it to the plasma membrane. To gain further insight into the potential mechanism through which SOS5 acts, localization of the SOS5 protein was examined using a reporter protein. SOS5 was cloned under the control of the UBIQUITIN 10 promoter, in a translational fusion with the mCitrine fluorescent protein. proUBQ10-SOS5$m$ Citrine plants fully complemented the sos5-2 mucilage adhesion phenotype, indicating that this fusion protein is functional (Figures 7A-C). Next we examined the localization of this protein in developing seeds at approximately 10 days postanthesis (DPA), when mucilage polysaccharides are being actively secreted into the apoplast of seed coat epidermal cells. While we cannot eliminate the possibility that the mCitrine molecule is cleaved from SOS5, the SOS5-mCitrine signal was predominantly detected with uniform labeling throughout the polysaccharides present in the mucilage pocket (Figures 7D,E). SOS5-mCitrine was also observed at the plasma membrane, as demonstrated by colocalization with the plasma membrane dye FM4-64. These results confirm the predicted SOS5 plasma membrane localization and previous observations of SOS5 being localized to the plasma membrane (Shi et al., 2003), and also show that in seed coat epidermal cells the majority of the protein is localized in the apoplast where it would be associated with mucilage polysaccharides.

\section{DISCUSSION}

In Arabidopsis, seed mucilage forms two discrete layers and studies of how these are formed are providing valuable information concerning our basic understanding of polysaccharide interactions. Here, we expand our knowledge of the mechanisms involved in mucilage adherence and further define two unique pathways that mediate polysaccharide interactions. Based on mutant mucilage phenotypes and double mutant analysis, CESA5 and MUM5 define one pathway that requires cellulose and hemicelluloses for mucilage adherence (Figure 1). A second pathway involving SOS5 and FEI2 functions independently of the CESA5-MUM5 pathway to mediate adherence. Single mutants can be divided into two distinct classes based on cellulose staining, one comprising cesa 5 and mum5 where rays of cellulose are intact yet diffuse staining cellulose is lost, and a second class including $\operatorname{sos} 5$ and fei2 where rays fail to form, while diffusely stained cellulose remains (Figure 1). We show that neither SOS5 nor FEI2 play a significant role in crystalline cellulose formation within mucilage (Figure 2). Finally, despite the similarities in the phenotypes of sos 5 and fei2 seeds, we identify some clear differences in mutant phenotypes that suggest that FEI2 has a role in cell wall organization beyond that mediated by SOS5 (Figures 4-6).

\section{Two Distinct Pathways Mediate Mucilage Adherence}

Previously, CESA5 and SOS5 had been shown to function independently in mucilage adherence (Griffiths et al., 2014). Here, we have expanded this analysis to include other genes that affect mucilage partitioning into layers, and define two distinct pathways that control adherence (Figure 8). The first pathway 
TABLE 2 | Macromolecular characteristics of wild-type Col-0, cesa5-2, sos5-2, and fei2-3 water-extracted mucilage.

\begin{tabular}{|c|c|c|c|c|c|}
\hline & RI Area & $M_{w}(k D)$ & $M_{n}(k D)$ & $M_{w} / M_{n}$ & {$[\eta](d L / g)$} \\
\hline \multicolumn{6}{|c|}{ First Polymeric Fraction } \\
\hline Wild-type & $0.8425(0.13)$ & $22717(826)$ & $22325(951)$ & $1.02(0.007)$ & $7.88(0.14)$ \\
\hline $\operatorname{sos} 5-2$ & $2.16(0.13)$ & $18415(600)$ & $16535(886)$ & $1.12(0.027)$ & $6.90(0.15)$ \\
\hline fei2-3 & $1.61(0.29)$ & $17253(840)$ & $15540(1156)$ & $1.11(0.029)$ & $7.24(0.06)$ \\
\hline \multicolumn{6}{|c|}{ Second Polymeric Fraction } \\
\hline Wild-type & $32.695(0.76)$ & $581(7.5)$ & 554 (6.9) & $1.05(0.002)$ & $5.96(0.08)$ \\
\hline cesa5-2 & $44.39(2.55)$ & $429(8.7)$ & $415(9.3)$ & $1.03(0.003)^{*}$ & $5.79(0.06)$ \\
\hline $\operatorname{sos} 5-2$ & 41.99 (1.52) & $569(5.1)$ & $537(5.6)$ & $1.06(0.06)$ & $5.71(0.06)^{*}$ \\
\hline fei2-3 & $38.26(4.75)$ & $541(5.0)$ & $519(6.7)$ & $1.04(0.004)$ & $5.91(0.07)$ \\
\hline
\end{tabular}

Values in parentheses are SE values of three biological replicates. $M_{\mathrm{w}}$, weight-average molar mass; $M_{\mathrm{n}}$, number-average molecular weight, $M_{\mathrm{w}} / M_{\mathrm{n}}$, polydispersity index, [ ], number-average intrinsic viscosity. Asterisk indicates a significant different value from wild-type $p<0.05$, bold values indicate a significantly different value from wild-type $p<0.001$; Mann-Whitney U test.

involves cellulose that is connected to the major component of mucilage, the pectin RG-I, through xylan branches synthesized by MUM5/MUCI21 (Harpaz-Saad et al., 2011; Mendu et al., 2011; Sullivan et al., 2011; Voiniciuc et al., 2015b; Ralet et al., 2016). Mutations in both of these genes produce seeds with similarly reduced mucilage adherence associated with the loss of the diffusely stained cellulose, while rays of cellulose remain above the columella (Figure 1), (Mendu et al., 2011; Sullivan et al., 2011; Voiniciuc et al., 2015b; Ralet et al., 2016). Furthermore, seeds from the cesa 5 mum5 double mutant did not show an additive phenotype confirming that the RG-I is attached through xylan branches to CESA5 synthesized cellulose. Furthermore, waterextracted mucilage from both single mutants does not contain the fraction of high molecular weight polymers, indicating that cellulose and hemicelluloses are required for the formation of these polymer aggregates in mucilage (Figure 6; Table 2), (Sullivan et al., 2011; Ralet et al., 2016). This pathway is also likely to involve IRX7 and IRX14, which are required for xylan biosynthesis and mucilage adherence, CESA3, and COBRALIKE 2 (COBL2) as cobl2 seeds have similarly reduced mucilage adherence while cellulose rays are still present (Ben-Tov et al., 2015; Griffiths et al., 2015; Voiniciuc et al., 2015b; Hu et al., 2016a,b).

The second pathway requires both SOS5 and FEI2, and affects mucilage adherence independently of CESA5 and MUM5/MUCI21. SOS5 appears to have lesser affects on mucilage adherence and cellulose crystallinity compared to FEI2 (Figures 1, 3, and 4), (Griffiths et al., 2014). The modest reduction in birefringence by sos5-2 mucilage was coherent with the weaker effect on mucilage partitioning between inner and outer mucilage layers, but was unexpected given that cellulose staining itself appeared to be reduced in a similar manner to that of fei2-3 mucilage. A sos5 mum5 double mutant showed a similar additive phenotype to that previously described for cesa 5 sos5, as did a cesa 5 fei2 double mutant (Figures 1 and 2), (Griffiths et al., 2014) demonstrating that neither SOS5 nor FEI2 affect mucilage adherence through the pathway involving RGI attachment to cellulose through xylan synthesized by MUM5 (Figure 1). Instead, SOS5 and FEI2 appear to be required for the adherence of mucilage through a mechanism that also involves the organization of cellulose into rays.
While these two independent pathways are clearly defined, we know far more about the role of cellulose in mediating adherence than we do of the role of SOS5-FEI2. It is curious that as many as six unique genes have been implicated in cellulose mediated mucilage adherence, while only SOS5 and FEI2 function have been identified in the alternative pathway. Other CESAs must be involved in the synthesis of the ray component of mucilage, and it is still possible that SOS5 or FEI2 could interact with other CESAs. Interestingly, heteromannans synthesized by CSLA2 and MUCI10 do not appear to fit into either pathway as LM21 labeling was still observed within cesa5, fei2, and sos 5 mucilage (Figure 3). Furthermore, there is more pectin than wild-type in the adherent mucilage layer of csla2 and muci10 seeds (Yu et al., 2014; Voiniciuc et al., 2015a). The increased labeling of cesa5 mucilage with LM21 is most likely due to increased accessibility to LM21 epitopes, potentially because there is less cellulose for heteromannans to bind (Figure 3).

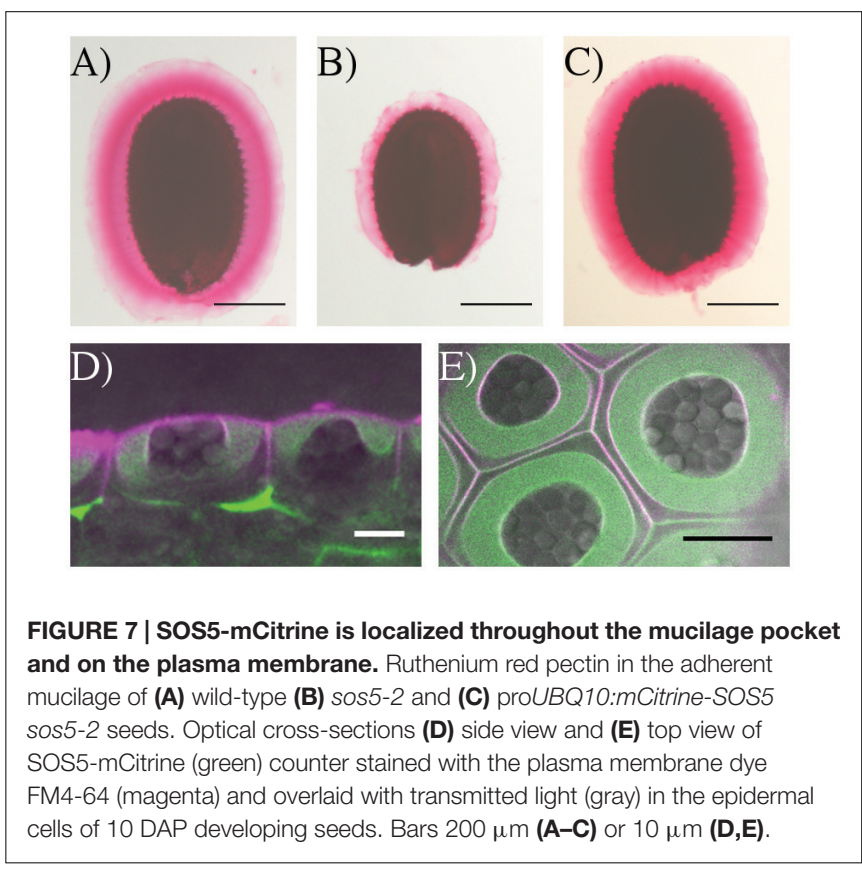




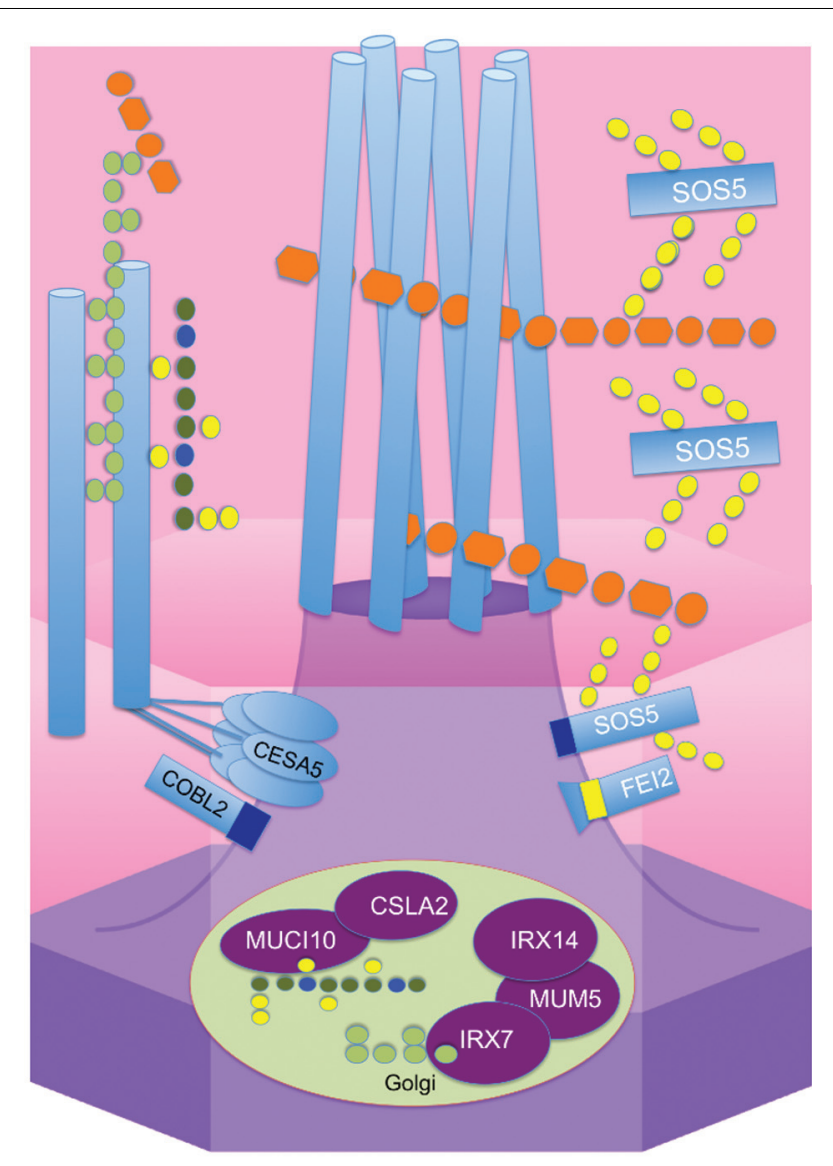

\section{Legend:}

\section{Cellulose}

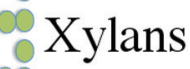

$:$

\section{Galactoglucomannan}

\section{Rhamnogalacturonan I}

\section{Galactan}

FIGURE 8 | Schematic model of the two distinct pathways that mediate mucilage adherence. CESA5 synthesizes cellulose which is connected to xylan branches synthesized by MUM5, IRX14, and IRX7 (Voiniciuc et al., 2015b; Hu et al., 2016a,b). Xylans are directly linked to pectin RG-I and can bind it to cellulose (Ralet et al., 2016). A second independent pathway mediating adherence requires SOS5 and FEI2 and is specifically required for ray formation, potentially through RG-I (Griffiths et al., 2014, this study).

LM14 immunolabeling of adherent mucilage suggests that other arabinogalactan proteins are involved in ray organization and mucilage adherence in addition to SOS5. Further genetic analysis using double mutant combinations of cesa5, sos5, or fei2 with csla2, muci10 or other arabinogalactan proteins expressed in the seed coat would help to further define these two pathways mediating mucilage adherence.

\section{Function of SOS5}

Salt-overly sensitive 5 was initially hypothesized to mediate cell adhesion through pectin in the cell wall as the fasciclin domains it contains are found in animal cell adhesion proteins (Shi et al., 2003). Subsequently, SOS5 was proposed to mediate cellulose biosynthesis through CESA5 due to the similar reduction in adherent mucilage observed in mutants affected in these two genes (Harpaz-Saad et al., 2011). This has since been negated, as cesa 5 and sos 5 have clearly different phenotypes with cellulose production apparently unaffected in sos5 (Griffiths et al., 2014). More recently, SOS5 was proposed to function in signaling pathways that mediate cell expansion (Seifert et al., 2014; Xue and Seifert, 2015). Determining the precise mechanism of SOS5 function is, therefore, proving to be difficult. Here, we confirm that cellulose biosynthesis is largely unaffected in sos 5 mucilage (Figure 2). Additionally, we have identified a key difference between sos 5 and both cesa 5 and fei2 as the adherent mucilage layer of sos 5 seeds contains more polysaccharide than either of the other mutants (Figures 4A,D). This increase in adherent mucilage is completely abolished on RGase treatment, indicating that it is mainly due to the amount of pectin, suggesting that SOS5 plays a relatively minor role in RG-I adherence compared to CESA5 and FEI2 (Figure 4). Finally, we have demonstrated that SOS5 is localized throughout the mucilage pocket as well as the plasma membrane in seed coat epidermal cells (Figure 7) suggesting that SOS5 may function at least partly in the apoplast in accord with a principal role in mediating pectin organization rather than cellulose biosynthesis or hormonal signaling. A structural role for SOS5 in organizing pectin is also supported by the modifications observed in the macromolecular properties of water-extracted mucilage polymers in sos5. Although sos 5 seeds have a similar composition to wild-type seeds, the polymers that form sos5 mucilage were smaller in both polymeric fractions obtained by SEC. Furthermore, the polymers in sos 5 water-extracted mucilage were significantly different from cesa5 mucilage, as they still contained the first very high-molecular weight population. This implicates SOS5 in the interactions between polysaccharides in mucilage, albeit independently of cellulose synthesized by CESA5 and hemicellulose synthesized by MUM5 and IRX14. Xylan has been shown to constitute a major connection between cellulose and mucilage RG-I (Ralet et al., 2016), while mutant phenotypes (Figure 4) indicate that SOS5 plays a lesser role in connecting these components. SOS5 could potentially organize the adherent mucilage halo through galactoglucomannans, which also play a role in mucilage organization and cellulose distribution (Voiniciuc et al., 2015a). Immunolabeling of heteromannans did not, however, reveal modifications to their distribution in the absence of SOS5, nor major changes in the intensity of their labeling (Figure 3) 
indicating that SOS5 is not required for their interactions within mucilage.

While the rays of cellulose are involved in mucilage expansion (Griffiths et al., 2015), how these are organized in the mucilage pocket prior to expansion remains to be determined. SOS5 could indirectly contribute to the orientation of cellulose microfibrils in the apoplast, or in the connections between cellulose and pectin. Other arabinogalactan proteins have been shown to be directly connected to pectin and hemicelluloses, forming a complex network (Tan et al., 2013), and we favor a similar hypothesis for the function of SOS5. Nevertheless, as indicated above the characteristics of water-extracted mucilage polymers from sos5, cesa5, and mum5 (Figure 5), and other mutant phenotypes exclude SOS5 from acting via cellulose, either directly or indirectly through hemicelluloses. While we cannot exclude the possibility that SOS5 binds directly to cellulose or glucans in the ray structure, given all the evidence, we propose that SOS5 is involved in organizing the ray through a few key connections between RG-I polysaccharides, and potentially organizing the cell wall through specific localization or anchoring of pectin components. Loss of SOS5 therefore results in fewer connections between pectin, and slightly reduced polymer size, but also loss of cell wall organization and ray formation. Galactan side chains are required for SOS5 function (Basu et al., 2015), and determining what role they play in mucilage adherence and cell wall organization, and if the FAS protein-protein interaction domains are important for this role are important questions. Characterizing the glycosyl side chain composition of SOS5, examining the binding affinity of SOS5 to cell wall polymers and testing potential protein-protein interactions with FEI2 or other factors should help to clarify its function.

\section{Function of FEl2}

FEI2 was proposed to control cellulose biosynthesis with this function being redundant with FEI1 in roots (Xu et al., 2008). Multiple reports have also implicated FEI2 as a key component of a signaling pathway that can monitor cell wall conditions, and initiate a cytosolic response, specifically altering cellulose biosynthesis (Xu et al., 2008; Steinwand et al., 2014; Basu et al., 2016). Yet the evidence supporting a role for FEI2 in cellulose biosynthesis is not convincing. Both cesa $6^{p r c 1}$ and cobra1 (cob1) mutants can enhance the root phenotype of feil fei2 mutants, suggesting that FEI1 and FEI2 function independently of these key components of cellulose biosynthesis (Xu et al., 2008). Here, we show that a fei 2 cesa 5 double mutant is more severe than either single mutant, similar to the cesa 5 sos 5 double mutant (Figure 1), (Griffiths et al., 2014), again suggesting that FEI2 functions independently of cellulose biosynthesis. The only evidence that FEI2 is involved in cellulose biosynthesis is the reduced amount of carbon incorporated into roots that have expansion defects (Xu et al., 2008). This could be a pleiotropic effect due to the inability of cells to expand, and not directly linked to cellulose biosynthesis ( $\mathrm{Xu}$ et al., 2008).

Here, comparison of the phenotypes of sos 5 and fei2 mucilage indicated that $\operatorname{sos} 5$ phenotypes are weaker than those of fei2
(Tables 1 and 2; Figure 4). Nonetheless, genetic analysis clearly supports a common pathway for mucilage organization involving SOS5 and FEI2 (Figure 1). This implies that FEI2 has some additional functions to those involving SOS5. FEI2 mutation had a stronger effect on crystalline cellulose amounts in the adherent mucilage layer, yet this reduction was not as severe as that observed for cesa5 and is independent of cesa5. FEI2 has previously been localized to the plasma membrane (Xu et al., 2008) and the localization observed for SOS5 suggests a common function at the plasma membrane.

Both SOS5 and FEI2 have been linked to hormone signaling, either through auxin, ethylene, or abscisic acid (Xu et al., 2008; Seifert et al., 2014; Steinwand et al., 2014; Xue and Seifert, 2015). A role in cell wall recognition and signaling remain the primary hypothesis explaining the function of FEI2, although the modified mucilage partitioning observed in sos 5 and fei2 is unconditional, unlike in roots where high salt concentrations are required for the phenotype to be observed. Despite this, both sos 5 and feil fei2 can enhance the root growth phenotype of untreated cob1 and prc1 mutants, suggesting this phenotype is not conditional (Xu et al., 2008). These results argue against a role for either SOS5 or FEI2 in signaling, especially in mucilage as the phenotypes are manifested in the absence of salt treatments. IAAALANINE RESISTANT 4 (IAR4) is involved in auxin signaling and maintaining auxin homeostasis, and iar 4 mutants can restore the feil fei2 root phenotype (LeClere et al., 2004; Steinwand et al., 2014). Nonetheless, iar4 cannot restore the mucilage defect of fei2 (Steinwand et al., 2014). We propose that SOS5 is required for interactions between cell wall components, which entail its correct localization within the apoplast, and that instead of a hormone-specific role, FEI2 would localize SOS5 in discreet regions in the plasma membrane. This hypothesis is consistent with the observation that SOS5 and FEI2 are required for the organization of cellulose into rays within mucilage and do not affect cellulose biosynthesis per se (Griffiths et al., 2014). FEI2 was previously proposed to localize cell wall biosynthetic machinery to a particular area of the plasma membrane (Xu et al., 2008), and as part of this mechanism, we hypothesize that this localization is vital for the function of SOS5.

In summary, here we further define two distinct pathways mediating mucilage adherence, one controlled by CESA5/MUM5, and another controlled by SOS5/FEI2. The cesa 5 mum 5 phenotype demonstrates genetically that cellulose synthesized by CESA5 and xylans synthesized by MUM5 interact to mediate RG-I mucilage adherence, consistent with biochemical data (Voiniciuc et al., 2015a; Ralet et al., 2016). We have identified additional differences between the mutant phenotypes of cesa5 and sos5/fei2, most notably the presence of crystalline cellulose in the adherent mucilage halo of sos5, and large molecular weight polymers present in sos5 and fei2 mucilage that are absent from cesa 5 mucilage. Finally, we have highlighted some minor differences in the phenotypes of sos 5 and fei 2 seeds, suggesting additional functions for FEI2 beyond the SOS5-FEI2 pathway. We demonstrate that SOS5 is localized throughout the mucilage pocket, consistent with a role of SOS5 in organizing the pectin component of mucilage around cellulose microfibrils that are required for ray formation. 


\section{AUTHOR CONTRIBUTIONS}

JG and HN conceived experiments. JG, M-JC, HN, M-CR, and GS performed the experiments JG, M-CR, and $\mathrm{HN}$ analyzed the data, JG wrote and $\mathrm{HN}$ revised the manuscript with critical reading from $\mathrm{M}-\mathrm{CR}$ and GS.

\section{FUNDING}

JG received the support of the EU in the framework of the Marie-Curie FP7 COFUND People Programme, through the award of an Agreenskills fellowship under grant agreement $\mathrm{n}^{\circ}$ 267196. The IJPB benefits from the support of the LabEx Saclay Plant Sciences-SPS (ANR-10-LABX-0040-SPS). GS was

\section{REFERENCES}

Alonso, J. M., Stepanova, A., N. Leisse, T. J., Kim, C. J., Chen, H., Shinn, P., et al. (2003). Genome-wide insertional mutagenesis of Arabidopsis thaliana. Science 301, 653-657. doi: 10.1126/science.1086391

Basu, D., Tian, L., Debrosse, T., Poirer, E., Emch, K., Herock, H., et al. (2016). Glycosylation of a fasciclin-like arabinogalactan-protein (SOS5) mediates root growth and seed mucilage adherence via a cell wall receptor-like kinase (FEI1/FEI2) pathway in Arabidopsis. PLoS ONE 11:e0145092. doi: 10.1371/journal.pone.0145092

Basu, D., Wang, M., Ma, S., DeBrosse, T., Poirier, E., Emch, K., et al. (2015). Two hydroxyproline galactosylatransferases, GALT5 and GALT2, function in arabinogalactan-protein glycosylation, growth and development in Arabidopsis. PLoS ONE 10:e0125624. doi: 10.1371/journal.pone.0125624

Ben-Tov, D., Abraham, Y., Stav, S., Thompson, K., Loraine, A., Elbaum, R., et al. (2015). COBRA-LIKE2, a member of the glycosylphosphatidylinositolanchored COBRA-LIKE family, plays a role in cellulose deposition in Arabidopsis seed coat mucilage secretory cells. Plant Physiol. 167, 711-724. doi: 10.1104/pp.114.240671

Blakeney, A. B., Harris, P. J., Henry, R. J., and Stone, B. A. (1983). A simple and rapid preparation of alditol acetates for monosaccharide analysis. Carbohydr. Res. 113, 291-299. doi: 10.1016/0008-6215(83)88244-5

Cosgrove, D. J. (2014). Re-constructing our models of cellulose and primary cell wall assembly. Curr. Opin. Plant Biol. 22, 122-131. doi: 10.1016/j.pbi.2014.11.001

Griffiths, J. S., Šola, K., Kushwaha, R., Lam, P., Tateno, M., Young, R., et al. (2015). Unidirectional movement of cellulose synthase complexes in Arabidopsis seed coat epidermal cells deposit cellulose involved in mucilage extrusion, adherence, and ray formation. Plant Physiol. 168, 502-520. doi: 10.1104/pp.15.00478

Griffiths, J. S., Tsai, A. Y.-L., Xue, H., Voiniciuc, C., Šola, K., Seifert, G. J., et al. (2014). SOS5 mediates Arabidopsis seed coat mucilage adherence and organization through pectins. Plant Physiol. 165, 991-1004. doi: 10.1104/pp.114.239400

Harpaz-Saad, S., McFarlane, H. E., Xu, S., Divi, U. K., Forward, B., Western, T. L., et al. (2011). Cellulose synthesis via the FEI2 RLK/SOS5 pathway and cellulose synthase 5 is required for the structure of seed coat mucilage in Arabidopsis. Plant J. 68, 941-953. doi: 10.1111/j.1365-313X.2011.04760.x

Haughn, G., and Chaudhury, A. (2005). Genetic analysis of seed coat development in Arabidopsis. Trends Plant Sci. 10, 472-477. doi: 10.1016/j.tplants.2005.08.005

Hellens, R. P., Edwards, E. A., Leyland, N. R., Bean, S., and Mullineaux, P. M. (2000). pGreen: a versatile and flexible binary $\mathrm{Ti}$ vector for Agrobacterium-mediated plant transformation. Plant Mol. Biol. 42, 819-832. doi: 10.1023/A:1006496308160

Hematy, K., and Höfte, H. (2008). Novel receptor kinases involved in growth regulation. Curr. Opin. Plant Biol. 11, 321-328. doi: 10.1016/j.pbi.2008.02.008

Höfte, H. (2015). The yin and yang of cell wall integrity control: brassinosteroid and FERONIA signaling. Plant Cell Physiol. 56, 224-231. doi: 10.1093/pcp/pcu182 supported by the Austrian Science Fund FWF grant number I1182-B22.

\section{ACKNOWLEDGMENT}

The authors wish to acknowledge the help of the glasshouse staff at INRA IJPB, and Pierre Briozzo for use of the polarizing lens.

\section{SUPPLEMENTARY MATERIAL}

The Supplementary Material for this article can be found online at: http://journal.frontiersin.org/article/10.3389/fpls.2016.01073

Hu, R., Li, J., Wang, X., Zhao, X., Wang, Z., Yang, X., et al. (2016a). Xylan synthesized by Irregular Xylem 14 (IRX14) maintains the structure of seed coat mucilage in Arabidopsis. J. Exp. Bot. 67, 1243-1257. doi: 10.1093/jxb/e rv510

Hu, R., Li, J., Xuanwen, Y., Zhao, X., Wang, X., Tang, Q., et al. (2016b). Irregular xylem 7 (IRX7) is required for anchoring seed coat mucilage in Arabidopsis. Plant Mol. Biol. doi: 10.1007/s11103-016-0493-4 [Epub ahead of print].

Johnson, K. L., Jones, B. J., Bacic, A., and Schultz, C. J. (2003). The fasciclinlike arabinogalactan protein family of Arabidopsis. A multigene family of putative cell adhesion molecules. Plant Physiol. 133, 1911-1925. doi: 10.1371/journal.pone.0025154

LeClere, S., Rampey, R. A., and Bartel, B. (2004). IAR4, a gene required for auxin conjugate sensitivity in Arabidopsis, encodes a pyruvate dehydrogenase E1 alpha homolog. Plant Physiol. 135, 989-999. doi: 10.1104/pp.104.040519

Macquet, A., Ralet, M. R., Kronenberger, J., Marion-Poll, A., and North, H. M. (2007). In situ, chemical and macromolecular study of the composition of Arabidopsis thaliana seed coat mucilage. Plant Cell Physiol. 48, 984-999. doi: 10.1093/pcp/pcm068

Marcus, S. E., Blake, A. W., Benians, T. A. S., Lee, K. J. D., Poyser, C., Donaldson, I., et al. (2010). Restricted access of proteins to mannan polysaccharides in intact plant cell walls. Plant J. 64, 191-203. doi: 10.1111/j.1365-313X.2010.04319.x

Mendu, V., Griffiths, J. S., Persson, S., Stork, J., Downie, A. B., Voiniciuc, C., et al. (2011). Subfunctionalization of cellulose synthases in seed coat epidermal cells mediates secondary radial wall synthesis and mucilage attachment. Plant Physiol. 157, 441-453. doi: 10.1104/pp.111.179069

Moller, I., Marcus, S. E., Haeger, A., Verhertbruggen, Y., Verhoef, R., Schols, H., et al. (2008). High-throughput screening of monoclonal antibodies against plant cell wall glycans by hierarchical clustering of their carbohydrate microarray binding profiles. Glycoconj. J. 25, 37-48. doi: 10.1007/s10719-007-9059-7

Nagaya, S., Kawamura, K., Shinmyo, A., and Kato, K. (2010). The HSP terminator of Arabidopsis thaliana increases gene expression in plant cells. Plant Cell Physiol. 51, 328-332. doi: 10.1093/pcp/pcp188

Ralet, M. C., Crepeau, M. J., Vigouroux, J., Tran, J., Berger, A., Salle, C., et al. (2016). Xylans provide the structural driving force for mucilage adhesion to the Arabidopsis seed coat. Plant Physiol. 171, 165-178. doi: 10.1104/pp.16. 00211

Seifert, G. J., and Roberts, K. (2007). The biology of arabinogalactan proteins. Annu. Rev. Plant Biol. 58, 137-161. doi: 10.1146/annurev.arplant.58.032806. 103801

Seifert, G. J., Xue, H., and Acet. T. (2014). The Arabidopsis thaliana FASCICLIN LIKE ARABINOGALACTAN PROTEIN 4 gene acts synergistically with abscisic acid signalling to control root growth. Ann. Bot. 114, 1125-1133. doi: 10.1093/aob/mcu010

Shaner, N. C., Steinbach, P. A., and Tsien, R. Y. (2005). A guide to choosing fluorescent proteins. Nat. Methods 2, 905-909. doi: 10.1038/nmeth819

Shi, H., Kim, Y., Guo, Y., Stevenson, B., and Zhu, J. (2003). The Arabidopsis SOS5 locus encodes a putative cell surface adhesion protein and is required for normal cell expansion. Plant Cell, 20, 3065-3079. doi: 10.1105/tpc.007872 
Steinwand, B. J., Xu, S., Polko, J. K., Doctor, S. M., Westafer, M., and Kieber, J. J. (2014). Alterations in auxin homeostasis suppress defects in cell wall function. PLoS ONE 9:e98193. doi: 10.1371/journal.pone.0098193

Sullivan, S., Ralet, M.C., Berger, A., Diatloff, E., Bischoff, V., Gonneau, M., et al. (2011). CESA5 is required for the synthesis of cellulose with a role in structuring the adherent mucilage of Arabidopsis seeds. Plant Physiol. 156, 1725-1739. doi: 10.1104/pp.111.179077

Tan, L., Eberhard, S., Pattathil, S., Warder, C., Glushka, J., Yuan, C., et al. (2013). An Arabidopsis cell wall proteoglycan consists of pectin and arabinoxylan covalently linked to an arabinogalactan protein. Plant Cell 25, 270-287. doi: 10.1105/tpc.112.107334

Thibault, J. F. (1979). Automatisation du dosage des substances pectiques par la method au metahydroxydiphenyle. Lebenssm. Wiss. Technol. 12, 247-251.

Tollier, M. T., and Robin, J. P. (1979). Adaptation de la method à l'orcinol sulfurique au dosage automatique des glucides neutres totaux: conditions d'application aux extraits d'origine végétale. Ann. Technol. Agric. 28, 1-15.

Voiniciuc, C., Gunl, M., Schmidt, M. H.W., and Usadel, B. (2015a). Highly branched xylan made by IRX14 and MUCI21 links mucilage to Arabidopsis seeds. Plant Physiol. 169, 2481-2495. doi: 10.1104/pp.15. 01441

Voiniciuc, C., Schmidt, M. H., Berger, A., Yang, B., Ebert, B., Scheller, H. V., et al. (2015b). MUCILAGE-RELATED10 produces galactoglucomannan that maintains pectin and cellulose architecture in Arabidopsis seed mucilage. Plant Physiol. 169, 403-420. doi: 10.1104/pp.15.00851
Wolf, S., Hematy, K., and Höfte, H. (2012). Growth control and cell wall signalling in plants. Annu. Rev. Plant Biol. 63, 381-407. doi: 10.1146/annurev-arplant042811105449

Xu, S. L., Rahman, A., Baskin, T. I., and Kieber, J. J. (2008). Two leucine-rich repeat receptor kinases mediate signalling, linking cell wall biosynthesis and ACC synthase in Arabidopsis. Plant Cell 20:3065-3079. doi: 10.1105/tpc.108.063354

Xue, H., and Seifert, G. J. (2015). FASCICLIN LIKE ARABINOGALACTAN PROTEIN 4 and RESPIRATORY BURST OXIDASE HOMOLOG D and F independently modulate abscisic acid signalling. Plant Signal. Behav.10:e989064. doi: 10.4161/15592324.2014.989064

Yu, L., Shi, D., Li, J., Kong, Y., Yu, Y., Chai, G., et al. (2014). CELLUlOSE SYNTHASE-LIKE A2, a glucomannan synthase, is involved in maintaining adherent mucilage structure in Arabidopsis seed. Plant Physiol. 164, 1842-1856. doi: $10.1104 /$ pp.114.236596

Conflict of Interest Statement: The authors declare that the research was conducted in the absence of any commercial or financial relationships that could be construed as a potential conflict of interest.

Copyright (c) 2016 Griffiths, Crepeau, Ralet, Seifert and North. This is an open-access article distributed under the terms of the Creative Commons Attribution License (CC BY). The use, distribution or reproduction in other forums is permitted, provided the original author(s) or licensor are credited and that the original publication in this journal is cited, in accordance with accepted academic practice. No use, distribution or reproduction is permitted which does not comply with these terms. 